Family Medicine and Community Health

\title{
The day the residents left: lessons learnt from COVID-19 for ambulatory clinics
}

\author{
Benjamin R Doolittle (D , ${ }^{1}$ Bradley Richards, ${ }^{2}$ Amerisa Tarabar $^{2}$ Matthew Ellman, ${ }^{2}$ \\ Daniel Tobin ${ }^{2}$
}

To cite: Doolittle BR, Richards B, Tarabar A, et al. The day the residents left: lessons learnt from COVID-19 for ambulatory clinics. Fam Med Com Health 2020;8:e000513. doi:10.1136/ fmch-2020-000513
Check for updates

(C) Author(s) (or their employer(s)) 2020. Re-use permitted under CC BY-NC. No commercial re-use. See rights and permissions. Published by BMJ.

${ }^{1}$ Department of Internal Medicine \& Pediatrics, Yale University, New Haven, Connecticut, USA

${ }^{2}$ Department of Internal Medicine, Yale University, New Haven, Connecticut, USA

Correspondence to Dr Benjamin R Doolittle; benjamin.doolittle@yale.edu

\section{ABSTRACT}

As the COVID-19 pandemic began, the residents from our ambulatory clinics were pulled to cover the increasing numbers of hospitalised patients. To provide care for our 40000 patients, without resident support, we needed to develop quickly a new culture of communication and innovation. We accomplished this by regular, transparent meetings with senior leadership and key stakeholders who were empowered to make rapid decisions. We then convened regular meetings with clinic leadership and frontline providers to receive feedback and implement new practices. These rapid meeting cycles allowed for a nimble response to a changing landscape. We optimised our video-conferencing and telehealth services, reached out to our most vulnerable patients and engaged other providers and medical students who were not engaged in patient care due to social isolation practices. We discuss the implications of these innovations on our future practice.

On 16 March 2020, we pulled our residents from clinic. The COVID-19 pandemic was coming to New Haven, Connecticut-a 2-hour drive from the New York City epicentre. Our residents were needed to staff makeshift intensive care units (ICUs) and COVID-19only wards. The patients were coming. By 21 April, our peak, 450 COVID-19 positive patients were in the hospital, in which 75 were intubated. Every resident and most faculty were drafted to care for these patients. While the news media rightly touted the sacrifice of heroes on the frontlines, there is another overlooked frontline: our ambulatory clinics.

Our ambulatory clinics care for more than 40000 active patients, most of whom are on state-funded insurance programmes in resource poor settings. With so many physicians deployed to inpatient services, a skeleton ambulatory crew was challenged to provide ongoing care. Due to necessary social isolation practices, our clinics severely restricted routine visits. The stakes were high. At our best, we struggled to mobilise the resources to support this vulnerable patient population. Amid the COVID-19 crisis, our aspirational medical home was inviable; we needed to refocus on survival. How do we provide quality care with a legion of chronically ill patients unable to see their physicians? The COVID-19 pandemic forced us to transform our ambulatory practice quickly. We did so through an emphasis on communication, innovation and technology.

\section{COMMUNICATION: 'TOGETHER, UNSTOPPABLE'}

One week before the first patient with COVID-19 was admitted to our hospital, our department and section leadership implemented mandatory, daily 'all-hands-on-deck' virtual meetings. Seven days a week, more than 60 clinical, educational, administrative and research leaders participated via video conferencing to debrief and troubleshoot emerging challenges. There was much to do: staff new clinical units, restructure inpatient and ambulatory teams, reconfigure educational activities, share administrative best practices, track patient flow and supply chain needs. Situational awareness and coordinated communication between the inpatient and ambulatory teams were essential.

Each meeting began with a daily assessment of the health and well-being of our personnel, followed by structured brief reports to keep us on track. This daily communicationfollowed by broadly disseminated email summaries-clarified the most pressing issues and helped us to forecast evolving needs. Furthermore, this open, daily communication promoted a corps d'esprit that we were in this together, that our leadership had our backs and that we were committed to a shared purpose.

Simultaneously, the health system launched various COVID-19 clinical task forces, including groups dedicated to ambulatory care. These teams coordinated all the diverse aspects of the clinical practices. Each workgroup had at least one representative on every other team, and decisions were communicated broadly through an official incident command centre structure. Clinic director involvement was required, and the traditional 
boundaries between the medical school and the hospital seemed to dissolve.

These approaches established a culture of accountability, an expectation of transparency and a spirit of rapid innovation. Communication that began early and occurred daily fostered a culture of action rather than reaction, even amidst uncertainty. Open display of realtime data and evolving protocols made for high value discussion. Teams were empowered to make decisions, which gave participants a sense of higher purpose and meaningful engagement. The tone set by our leadership of shared vision, respect and optimism made our new department motto have real meaning: 'Together. Unstoppable.'.

\section{INNOVATION AND TECHNOLOGY}

After implementing a new model of communication, we were faced with the immediate challenge of managing 40 000 patients remotely across several clinics, many of whom were clinically complex and socially vulnerable. The need was urgent, and we knew that we could not accomplish the task with our existing resources and workflows. To overcome this obstacle, we needed to think creatively, tap into non-traditional resources, and leverage technology to bridge the physical divide between our providers and socially distanced patients.

Transitioning our visits-more than 400 per day-to remote care represented our most pressing task. To accomplish this, we needed to rapidly implement telehealth technology—a long-anticipated but profoundly underdeveloped institutional resource. This entailed recruiting as many patients as possible to sign-up for the embedded patient portal (MyChart) in Epic, our electronic medical record (EMR). This would allow them to participate in video visits using a system-approved, Health Insurance Portability and Accountability Act (HIPAA) compliant platform. A clunky registration process and lack of access to broadband internet were long-standing barriers for many of our patients. Fortunately, we recruited a team of volunteer medical students and medical assistants to launch a campaign to increase utilisation. MyChart and video visit access improved dramatically, and while largely successful, the inequity of resources continued to be a problem for many of our patients, of whom $60 \%$ earn less than 10000 dollars per year. ${ }^{1}$ We still relied heavily on audio-only technology (ie, telephone visits). Sadly, our innovative spirit could not fully overcome the regulatory barriers prohibiting the use of non-approved telehealth platforms that may have opened access for those patients unable to use the EMR technology. Even so, this effort helped us to accomplish in weeks a task that was planned to roll out over years. Remarkably, using telehealth, we reached $70 \%$ of our pre-COVID-19 visit volume.

Simultaneously, we developed new workflows to empower the few remaining ambulatory physicians to cover the tasks of those pulled to inpatient service. We asked all residents and faculty to grant EMR in-basket access to every other provider, had everyone scrub their schedules to determine who needed to be seen in person and directed each provider to create a list of patients most likely to decompensate without proactive care. Here, again, effective communication through our regular clinic meetings was essential and could only have been accomplished with the shared sense of trust, purpose and commitment engendered with our new communication protocols. We then leveraged the talent of our remaining ambulatory nursing staff to organise these lists within our EMR, reach out to patients and screen them for decompensating chronic disease. Similarly, our nurses were essential partners to help make sure refill requests, urgent paperwork and lab results were all addressed and that nothing fell through the cracks.

Importantly, we also developed a telehealth COVID-19 clinic. As the incidence of COVID-19 grew exponentially in our community, we realised that we required a way to track, monitor and proactively address the medical needs of these patients. Once again, we needed to use our resources creatively and leverage technology to help us address this challenge. We worked with our EMR physician champions and practice managers to develop daily reports of patients with COVID-19. Then, we recruited an interdisciplinary team of volunteer physician assistant faculty to implement a clinical monitoring protocol to screen patients for decompensation. This was work intensive and required both internal and external coordination between team members and the various clinical task forces to stay up to date with the latest testing and treatment algorithms. In the end, this programme monitored hundreds of patients which, we believe, helped save lives.

\section{THE FUTURE OF AMBULATORY CARE}

The day the residents left the clinic was a dark milestone. We were overwhelmed by critically ill patients suffering from a disease we were only beginning to understand. Abandoning the clinic to staff makeshift ICUs and COVID-19 care units was necessary, but it reflected the failure of our healthcare system to forestall the workforce demands of the pandemic. Our primary care centres, devoted to serving our most vulnerable patients, had become overwhelmed by the pandemic.

And yet, from the rubble of our broken system, we are building a new foundation. Social distancing and clinical isolation forced us to forge new connections in innovative ways. The silos we worked in evolved into coordinated teams. We pulled together to solve real problems. Inefficient and protracted group meetings were replaced by daily team huddles characterised by transparency, high yield information sharing and effective innovation. Unreliable transportation, costly parking and busy waiting rooms were exchanged for telecommuting and telehealth workflows that helped us stay on task and on time. We learnt to cut all the fat-and some of the bone-and run a lean clinical operation that painfully but effectively kept tabs on our most vulnerable patients 
and allowed us to care for them. We recruited interdisciplinary teams of volunteers to monitor our COVID-19 positive homebound patients throughout their illness and quickly mobilised advanced care at the first signs of clinical decompensation.

It is likely that the COVID-19 pandemic will continue to plague our patients and disrupt our ambulatory care practices in the months ahead. Although changing conditions on the ground will dictate the best response, our experience has taught us that adaptation and innovation under stress are both possible. To this end, we recommend the following. First, convene regular, transparent meetings between senior leadership and relevant clinic managers with broad representation. Situational awareness and the capacity to make decisions quickly were critical to our success. Face-to-face meetings may continue to be challenging, but video conferencing and robust use of information technology can facilitate communication between teams. Second, replicate these meetings among frontline clinic staff and providers doing direct patient care to allow for rapid implementation and local troubleshooting. We recommend that all staff take turns chairing the meeting to encourage broad participation and buyin. Make sure to engage those staff that are isolating at home. Third, partner with those who are only indirectly involved with the pandemic response; our collaboration with physician assistant faculty and medical students to reach out to the most vulnerable of our patients was an important contribution. Finally, we encourage health systems to foster a spirit of innovation that solicits creative approaches to problem solving.

The cost of this pandemic is incalculable, both in human lives as well as dollars, but we persevere, innovate and even thrive. A new clinical model is emerging, and telemedicine will persist in our new reality. Must homebound patients come for an in-person visit every time? Both patients and physicians might prefer more regular check-ins with telehealth. ${ }^{23}$ We must find the balance between the efficiency and accessibility of telemedicine without abandoning the need to sit across from our patients and connect face to face. We must recognise that medical care exists on an ever-changing continuum between the inpatient and outpatient settings and stay flexible enough to enhance the care in one setting without abandoning the other. We must preserve the spirit of innovation, the collaboration across silos and the sense of urgency and model this for our trainees. When we welcome the residents back, let us invite them to rejoin us in a house rebuilt.

Contributors All authors participated in the preparation of the manuscript. This paper is not under consideration elsewhere and has not been previously published.

Funding The authors have not declared a specific grant for this research from any funding agency in the public, commercial or not-for-profit sectors.

Competing interests None declared.

Patient consent for publication Not required.

Provenance and peer review Not commissioned; externally peer reviewed.

Open access This is an open access article distributed in accordance with the Creative Commons Attribution Non Commercial (CC BY-NC 4.0) license, which permits others to distribute, remix, adapt, build upon this work non-commercially, and license their derivative works on different terms, provided the original work is properly cited, appropriate credit is given, any changes made indicated, and the use is non-commercial. See: http://creativecommons.org/licenses/by-nc/4.0/.

ORCID iD

Benjamin R Doolittle http://orcid.org/0000-0002-6922-6556

\section{REFERENCES}

1 Doolittle B, Courtney M, Jasien J. Satisfaction with life, coping, and spirituality among urban families. J Prim Care Community Health 2015;6:256-9.

2 Becevic M, Boren S, Mutrux R, et al. User satisfaction with telehealth: study of patients, providers, and coordinators. Health Care Manag 2015;34:337-49.

3 Polinski JM, Barker T, Gagliano N, et al. Patients' satisfaction with and preference for telehealth visits. J Gen Intern Med 2016;31:269-75. 